\title{
Screening for abdominal aortic aneurysm reduced death from AAA in older men
}

The Multicentre Aneurysm Screening Study Group. The Multicentre Aneurysm Screening Study (MASS) into the effect of abdominal aortic aneurysm screening on mortality in men: a randomised controlled trial. Lancet 2002;360:1531-9.

\section{QUESTION: In older men, does ultrasonographic screening for abdominal aortic aneurysm (AAA) reduce death from AAA?}

\section{Design}

Randomised (allocation concealed*), blinded (data collectors and outcome assessors),* controlled trial with mean 4.1 year follow up (Multicentre Aneurysm Screening Study).

\section{Setting}

4 screening and 4 academic centres in England, UK.

\section{Patients}

67800 men who were $65-74$ years of age (mean age 69 y) and were identified from family physician and Health Authority patient lists. Family physicians excluded patients (without knowledge of randomisation) they considered unfit to be screened, including those with terminal illness, other serious health problems, or previous AAA repair. Mortality follow up was $99 \%$.

\section{Intervention}

Men were allocated to be invited $(n=33$ 839) or not invited ( $\mathrm{n}=33$ 961) for screening. The invited group received by post an invitation on the family physician's letterhead, an information booklet, and a questionnaire. Those accepting the invitation attended a clinic and had an ultrasonographic scan of the abdominal aorta. Scan results were sent to the family physicians. No contact was made with men in the uninvited group with respect to screening.

\section{Main outcome measures}

Death from AAA. Secondary outcomes were all cause mortality, ruptured AAA, and quality of life.

\section{Main results}

$80 \%$ of men in the invited group accepted the invitation and were scanned. Fewer deaths from AAA occurred in the invited than in the control group (table). Adjustment for patient age and screening centre did not affect the result. Ruptured AAA was also lower in the invited group (table). Groups did not differ for all cause mortality. Groups did not differ for anxiety, depression, or health status measures.

\section{Conclusion}

In older men, ultrasonographic screening for abdominal aortic aneurysm (AAA) reduced death from AAA without any detectable reduction in quality of life.

*See glossary.

\section{COMMENTARY}

In the study by the Multicentre Aneurysm Screening Study Group, ultrasonographic screening, compared with usual care, decreased aneurysm related mortality and aneurysm rupture. Causes of death were identified in the entire population, and compliance with the screening program was high. Operative mortality in this study was higher in the control than in the treatment group $(21 \% v 6.5 \%, \mathrm{p}<0.001)$, magnifying the benefit of screening. In addition, the results cannot be generalised to women or to men $>74$ years of age. In an older population, comorbid conditions compete for death and affect both the results of surgery (increased mortality) and the benefit of screening (reduced life expectancy).

Screening potentially prevents 14 aneurysm related deaths per 10000 patients over 4 years, which is a relatively small number compared with the total number of expected deaths (290 deaths per 10000 ). This suggests that screening is unlikely to have a substantial effect on the overall burden of deaths at a population level (about $1 \%$ of deaths/y in these moderately high risk patients) or at the individual patient level. The first reason is that not all deaths, even in patients with aneurysms, result from rupture. The second is related to Geoffrey Rose's preventive paradox. ${ }^{1}$ In a moderately high risk population, a large proportion will have only small to moderately sized aneurysms $(3-4.5 \mathrm{~cm})$. Theoretically, offering surgery to these patients would reduce a large number of deaths at a population level but slightly change the risk of death of each individual patient. In addition, surgery carries a substantial risk of mortality and morbidity and is resource intensive compared with other cardiovascular risk prevention strategies. While screening may be cost effective in the British nationalised health care system, ${ }^{2}$ it requires careful scrutiny and consideration of the large number of repeated ultrasonography scans for small $(3-4.5 \mathrm{~cm})$ aneurysms.

Until further research identifies patients at higher risk of aneurysm rupture, screening is acceptable, but not mandatory, in older men who are suitable candidates for surgery and who would undergo surgery were they found to have an aortic aneurysm $>5.5 \mathrm{~cm}$

Claudio S Cina, MD, MSc McMaster University, Hamilton, Ontario, Canada

1 Rose GA. The strategy of preventive medicine. Oxford: Oxford

2 Multicentre aneurysm screening study (MASS): cost effectiveness analysis of screening for abdominal aortic aneutrial. BMJ 2002;325:1135-8.

Invited $v$ not invited for abdominal aortic aneurysm (AAA) screening at mean 4.1 yearst

\begin{tabular}{lllll} 
Outcomes & Invited & Not invited & RRR ( $95 \% \mathrm{Cl})$ & NNS (Cl) \\
Death from AAA & $0.19 \%$ & $0.33 \%$ & $42 \%(22$ to 58$)$ & 712 (500 to 1000) \\
\hline $\begin{array}{c}\text { Nonfatal ruptured } \\
\begin{array}{l}\text { AAA plus AAA } \\
\text { related deaths }\end{array}\end{array}$ & $0.24 \%$ & $0.41 \%$ & $41 \%(23$ to 55$)$ & 589 (334 to 1000$)$ \\
\hline
\end{tabular}

†NNS = number needed to screen. Other abbreviations defined in glossary; RRR, NNS, and Cl calculated from data in article. 